\title{
Ueber die Verseifbarkeit einiger Säureimide (Diamide) und Aminsäuren durch Fermente.
}

Von

\section{Dr. M. Gomnermann.}

Den in diesem Archiv Bd. 89 S. 493 veröffentlichten Untersuchungen über die Verseifbarkeit einiger Säure-Amide und -Anilide durch Fermente reihen sich die nachstehenden als Ergänzung an; es kann daher von der Wiedergabe der allgemeinen Methode des Verfahrens abgesehen und auf die erste Arbeit verwiesen werden; nur die Abweichungen, welche die Imide (Diamide) bei den einzelnen Versuchen bedingen, sollten Erwähnung finden; bemerkt soll aber hier auch sogleich werden, dass ich die Unlöslichlzeit des Fluornatriums in $55 \%$ igem Alkohol, auf die ich in meiner ersten Versuchsreihe noch nicht aufmerksam geworden war, jetzt wiederholt zur Entfernung dieses oft störend wirkenden Stoffes aus den Versuchsgemischen benutzt habe.

Die verwendeten Fermente sind dieselben geblieben wie bei der ersten Versuchsreihe; die Organe - Leber und Niere - stammten gleichfalls vom Schaf. Die zur Prufung gelangten Imide und Aminsäuren waren folgende: Oxaminsäure, Succinimid, Succinaminsäure, Dibenzamid, Disalicylamid, Phtalimid, denen sich Parabansäure anschloss. Versuche mit Diacetamid und Oximid habe ich nicht ausgeführt, weil die Darstellung dieser beiden Präparate zu umständlich ist; bei dem Diacetamid ist zudem ohne Weiteres nach meinen Versuchsresultaten anzunehmen, dass dasselbe durch diejenigen Fermente zerlegt wird, die das Acetamid spalten $^{1}$ ). An Stelle des Oximids andererseits glaubte ich Oxamin-

1) Während diese Arbeit druckfertig war, kommt mir durch Prof. Nas se ein Abdruck eines Vortrages von F. Hofmeister: Ueber den Ban des Eiweissmolekuls, gehalten auf der 74. Versammlung deutscher Naturforscher und Aerzte in Karlsbad (Naturwissensch. Rundschau Bd. 17 Nr. 42 u. 43. 1902), zur Hand, 
säure setzen und im Falle der Nichtzersetzung durch Fermente und Histozyme auf ein gleiches Verhalten des Oximids selbst schliessen zu durfen.

Versuche uber die Zersetzung der hier in Frage kommenden Imide im Thierkörper sind nur von Fr. Koehn ${ }^{1}$ ) angestellt worden; bei dem Succinimid, Dibenzamid und Phthalimid wird der von Koehne erhaltenen Resultate gedacht werden.

Succinimid und Phthalimid waren von Kahlbaum-Berlin bezogen; die ủbrigen Präparate habe ich selbst dargestellt.

\section{0xaminsänre.}

Der Darstellung der Oxaminsäure lag zunáchst die Arbeit von Ost und Mente ${ }^{2}$ ) zu Grunde. Nach Balard ${ }^{3}$ ) soll das saure Ammoniumoxalat im Oelbad auf $220^{\circ}-230^{\circ}$ erhitzt werden, die Masse wird hierbei anfangs flussig, dann teigig und blaht sich schliesslich unter Entwicklung von Ammoncarbonat auf. Ost und Mente geben die Zersetzungstemperatur bei $140^{\circ}$ an, bis die Masse zähe wird, - es ist mir nicht gelungen, bei dieser Temperatur eine solche Masse zu erlangen, sondern dieser Zustand trat erst bei $180^{\circ}$ ein.

Nach dieser Vorsehrift arbeitend, musste bald auffallen, dass das oxaminsaure Baryum in Lösung bleiben soll, während zur Darstellung dieses Salzes oxaminsaures Ammonium durch Baryumchlorid umgesetzt wird, - d. h. oxaminsaures Baryum fällt aus ${ }^{4}$ ), wie denn auch die meisten Salze der Oxaminsaure sehr schwer in Wasser löslich $\sin d$.

In welchem es heisst: „Das auf Eiweiss so kraftıg wirkende Trypsin ist nucht im Stande, Hippursaure zu spalten oder auch nur aus sonst so labilen Saureamiden we Acetamid und Asparagin den Ammoniakrest abzutrennen." Ich habe daraufhin meine Versuche wiederholt und fand die Resultate der ersten Versuclisreihe voll bestatigt: Trypsin verseift Acetamid unter Abspaltung von Essigsaure; es entwickelte sich sogar beim Eindampfen des Hauptversuches Ammoniak, welches rothes Lackmuspapier blaute, wahrend im Controlversuch Acetamid sich verfluchtigt und blaues Lackmuspapier durch die Dampfe schwach gerothet wird.

1) Ueber das Verhalten einiger Saureimide $1 \mathrm{~m}$ thierischen Organismus. Aus dem Institut fur physiol. Chemie und Pharmakologie in Rostock (Director Prof. Dr. O. Nasse). Inaug.-Dissert. Rostock 1894.

2) Berichte d. chem. Gesellsch. Bd. 19 S. 3229.

3) Annal. f. Chem. Bd. 42 S. 197.

4) Engstrom, Annal. f. Chem. 1856 S. 453. 
Die Ausbeute war ùbrigens sehr gering.

Viel schneller und ganz glatt im Verlauf erhält man die Oxaminsäure nach Toussaint ${ }^{\mathbf{1}}$ ) aus Oxamid durch Kochen mit Ammoniak; nach vierstundigem Kochen war das Oxamid vollstảndig gelöst und, da beim Erkalten der Lösung keine Ausscheidung erfolgte, in Oxaminsäure ubergegangen; die heisse Lösung wurde filtriert und so lange eingedampft, bis beim Herausnehmen einer Probe dieselbe sofort erstarrte; dann wurde starke Salzsảure bis zur stark sauren Reaction unter Umrubren zugefügt und das Gemisch 24 Stunden stehen gelassen. Auf Zusatz der Salzsäure schied sich die Oxaminsaure sofort als weisses Pulver aus; die úberstehende Flüssigkeit wurde abgegossen, der Niederschlag mit alkoholhaltigem Wasser, welche zwar Salmiak, jedoch nicht Oxaminsaure lost, ausgesüsst und schliesslich auf dem Saugfilter mit verdüntem Alkohol ausgewaschen, bis Silberlosung keine Trubung bewirkte. Eine Probe des getrockneten, weissen Pulvers in heissem Wasser gelöst gab auf Zusatz von etwas Ammoniak durch Calciumchlorid keine Trubung; ebenso wurde Permanganatlösung bei Gegenwart von Schwefelsäure nach fünf Minuten nicht entfärbt, so dass ich wohl annehmen kann, dass die Oxaminsäure völlig frei von Oxalsäure war.

Die nun folgenden Versuche mit Fermenten lehnen sich ganz denen mit Oxamid an: von den Filtraten wurden $50 \mathrm{ccm}$, entsprechend 0,25 Oxaminsäure, abgenommen, durch Ammoniak alkalisch gemacht aufgekocht, nochmals filtrirt, das Filtrat siedendheiss mit Essigsaure übersattigt und noch heiss mit 10\% iger Calciumchloridlosung versetzt, ein entstehender Niederschlag mit heissem Wasser ausgewaschen, bis Ammoniumoxalat keine Trübung mehr gab, und auf Oxalsäure in stark schwefelsaurer Lósung durch Permanganat gepruft.

Versuche mit thierischen Fermenten.

Pepsin. Es wurden nur 1,6 cem Permanganatlosung verbraucht, und blieb die Farbe eine halbe Stunde unverändert, ganz gleich wie bei den Versuchen mit Oxamid; Oxaminsaure wird durch Pepsin nicht in Oxalsäure ubergefuhrt.

Trypsin. Die zugesetzten $25 \mathrm{ccm}$ Sodalösung wurden durch die Oxaminsäure völlig neutralisirt, so dass sich ein weiterer Zusatz

1) Annal. f. Chem. Bd. 120 S. 287. 
Ueber die Verseifbarkeit einger Saureimide (Diamide) u. Aminsauren etc. 281 von 25 cem nothig machte; eine Umsetzung in Oxalsảure fand nicht statt.

Ptyalin ist ohne Einwirkung.

Versuche mit Histozymen.

Leber wirkt nicht auf Oxaminsảure ein, wie daraus hervorgeht, dass der Verbrauch an Permanganat sebr gering und in beiden Versuchen ziemlich das Gleiche war; es mussten bei einer Umsetzung in Oxalsäure 0,3 Oxaminsäure - ich nehme an, dass ein Theil in dem Pressruckstand geblieben ist $-76 \mathrm{ccm} 1 / 10$ Permanganatlösung benöthigt haben.

Niere war gleichfalls wirkungslos.

Versuche mit pflanzlichen Fermenten.

Maltin und Invertin sind unwirksam.

Emulsin. Das Emulsin löste sich im Hauptversuch nicht auf, jedenfalls in Folge der sauren Reaction des Gemisches; nach zweitảgigem Stehen nahm ich die Prufung auf Oxalsäure vor, fand jedoch keine; ich fùgte somit einem neuen Versuch $30 \mathrm{ccm}$ Sodalósung hinzu, - das Emulsin blieb in truber Lösung, und auch in diesem Versuch war Oxalsäure nicht nachzuweisen.

Aus meinen Versuchen mit den einfachen Oxalsäure-Amiden geht hervor, dass dieselben durch alle angewandten Enzyme nicht gespalten werden, auch unzersetzt und, im chemischen Sinu, unschädlich den Thierkörper passiren, wie ich als sicher angenommen habe und durch Kobert's neue Versuche mit reinem Oxamid, wie er mir mittheilte, bestatigt worden ist. Anders dagegen steht es mit den complicirten Verbindungen - insbesondere Parabansäure, Alloxan und Alloxantin -, die nach den Versuchen von Koehne im Thierkorper fast vollkommen zerstört werden. Durch welche Kräfte dies geschieht, ergeben die anschliessenden Versuche; was das Wie angeht, so ist wohl mit Sicherheit anzunehmen, dass aus den genannten Körpern zunächst Oxalsäire abgespalten wird - bei der Parabansäure wahrscheinlich nach vorgangiger Umwandlung in Oxalursäure -, die denn, wie auch K o ehn e aus dem Nichtauftreten von Oxalursäure im Harn schliesst, sofort weiter zerfällt. Der Vollständigkeit wegen fuhrte ich einige Versuche mit Parabansaure $\mathrm{NH}\left\langle\begin{array}{l}\mathrm{CO}-\mathrm{CO} \\ \mathrm{CO}-\mathrm{NH}\end{array}\right.$ aus; diese geht sehr leicht unter Aufnahme von 
Wassermolekủlen in Oxalursäure und Oxalsäure über; es ist demnach eine Umsetzung durch Fermente leicht zu erkennen, wenn im Reactionsgemisch sich Oxalsäure nachweisen lässt. Koehne gibt als charakteristische Reaction fưr Parabansäure an - und bezieht sich auf "Harnanalyse" von Neubauer und Vogel und auch Beilstein -, dass ammoniakalische Chlorcalciumlósung in der Kälte keinen Niederschlag gibt; in der "Harnanalyse" ${ }^{1}$ ) finde ich jedoch folgende Angabe, die sich nicht auf Parabansảure, sondern auf Oxalursảure bezieht: „Versetzt man eine wảssrige, mässig verdunnte Lösung von oxalursaurem Ammon mit Chlorcalcium und Ammoniak, so entsteht kein Niederschlag; die Flússigkeit bleibt vollkommen klar; erwärmt man dagegen die Mischung, so tritt sehr bald, noch weit vor der Siedhitze, Trübung ein, und oxalsaurer Kalk scheidet sich massenhaft aus. Dieses Verhalten ist unzweifelhaft die empfindlichste Reaction, mit welcher sich noch unglaublich kleine Mengen von Oxalursáure erkennen lassen, sobald man das Mikroskop zu Hulfe nimmt." -

Nach diesen Angaben Neubauer's wurde also, falls in den Versuchsgemischen sich oxalsaurer Kalk ausscheidet, die Parabansäure bereits durch Einwirkung von Fermenten in Oxalursäure ủbergegangen sein. Wie äusserst schnell diese Umsetzung eintritt, ergeben die nachfolgenden Versuche.

Um nun aber sicher zu sein, dass die eventuell nachgewiesene Oxalsaure nur durch Einwirkung der Fermente entstanden ist, dampfte ich eine schwach durch Soda alkalisch gemachte Lósung von Parabansäure $(0,5: 100) \mathrm{Kahlbaum}$ auf dem Wasserbad zur Trockne ein, nahm das blendend weisse Pulver im Wasser auf, ủbersättigte die Lósung mit Salzsäure, fugte Ammoniak und Chlorcalciumlosung zu und beobachtete, dass sofort ein starker Niederschlag eintrat, welcher unter dem Mikroskop krystallinische Gebilde schwer erkennen liess; jedoch erschienen die charakteristischen Formen des Calciumoxalates, als der Niederschlag in etwas Salzsảure und Wasser gelost und Ammoniak vorsichtig ubergeschichtet wurde, - bis sich die Flüssigkeit vollstảndig geklärt hatte.

Nach diesem uberraschenden Resultat leitete ich folgende Versuche ein:

1. eine Lösung von Chlorcalcium, mit Ammoniak versetzt, wurde erwärmt und bei Seite gestellt;

1) Neubauer und Vogel S. 34. 
Ueber die Verseifbarkeit einiger Sảureimide (Diamide) u. Aminsanren etc. 283

2. eine Lösung von Parabansäure mit Ammoniak neutralisirt und s of o r t mit Chlorcalcium versetzt;

3. die gleiche Lösung drei Stunden stehen gelassen und dann mit Chlorcalcium versetzt;

4. die gleiche Lösung auf dem Wasserbad erwärmt, abgekühlt und derselben Chlorcalcium zugegeben;

5. eine Lösung von Parabansäure im Wasserbad von $60^{\circ}$ 1/2 Stunde erwärmt, dann Ammoniak und Chlorcalcium zugefügt und beobachtet, dass bei

1. keine Veränderung eintrat, da selbstverständlich;

2. nicht sofort ein Niederschlag entstand, jedoch nach schwachem Erwärmen;

3. ein Niederschlag nach einigen Secunden sich bildete;

4. der Niedersehlag sofort eintrat;

5. nicht sofort, jedoch nach kurzem Stehen bei Zimmertemperatur ein Niedersehlag entstand.

Die Niederschläge bestanden aus Calciumoxalat.

Nach diesen Versuchen wird somit die Parabansäure bei Gegenwart von Soda und Ammoniak selbst bei niedrigerer Temperatur, als sie beim Eindampfen auf dem Wasserbad erzielt wird, sowie bereits beim einfachen Erwärmen einer wảssrigen Losung derselben auf $60^{\circ} \mathrm{C}$. schon in Oxalsaure umgesetzt; da nun auch eine ammoniakalische Parabansảurelósung für sich bereits nach einigen Stunden verändert wird und mit Chlorealciumlósung einen Niederschlag von Calciumoxalat gibt, sämmtliche Versuchsflusssigkeiten aber, besonders diejenigen mit thierischen Organen, um die Eiweissstoffe zu entfernen, erwärmt oder aufgekocht werden mussen, so können die Resultate der Versuche uber die Einwirkung von Fermenten auf Parabansäure nur sehr zweifelhafter Natur sein, und babe ich aus diesem Grunde solche nicht ausgefuhrt; es lässt sich also leider experimentell nicht nachweisen, in welchen Organen die Bildung von Oxalursäure oder Oxalsäure aus Parabansäure vor sich geht, vielleicht schon im Magen durch die Salzsäure oder im alkalischen Dúnndarm?

\section{Succinimid.}

Bei diesen Versuchen machte ich anfangs die Erfahrung, dass bei allen Aetherausschuttelungen der Haupt- und Controlversuche. 
stets ein stickstoffhaltiger Rückstand erhalten wurde; es musste mich dies befremden, da bei dem Zusatz von Phosphorsäure etwa gebildete Succinaminsaiure in den Aether nicht ubergehen konnte, weil diese in Aether und Alkohol unloslich ist, und die erhaltenen Krystalle die rhombischen Tafeln des Succinimids zeigten. Es lag also die Möglichkeit nahe, dass das Succinimid sich schwerer in Aether und Alkohol löst, als in der Literatur angegeben ist, und daher der Verdampfungsrückstand nicht genugend mit Aetheralkohol ausgeschüttelt worden war.

Um die Gegenwart der Bernsteinsảure nachzuweisen, benutzte ich die Fällbarkeit von Eisenoxydlösungen durch ihre Neutralsalze in der Siedhitze; den vom Aetheralkohol ungelosten, von Succinimid vollig befreiten, getrockneten Ruckstand extrahirte ich mit $55 \%$ jgem Alkohol, welcher nach meinen bereits erwähnten Versuchen kaum eine Spur des sehr störend wirkenden Fluornatriums aufnimmt, dagegen Natriumsuccinat leicht löst, verdampfte das Filtrat zum Theil, neutralisirte und prufte mit Eisenchloridlösung: eine beim Erhitzen auftretende gallertartige Ausscheinung konnte nunmehr nur durch die Gegenwart von Bernsteinsäure bedingt sein, denn Succinaminsäure fảrbtzwar Eisenchloridlösung dunkel blutroth, Iasst jedoch kein basisches Salz beim Erhitzen ausfallen, und Succinimidgibt mit Eisenlösung überhaupt keine Farbenreaction.

Versuche mit thierischen Fermenten.

Pepsin. Das Versuchsgemisch wurde unter Zusatz von etwas Natriumbicarbonat zur Trockne eingedampft. Bernsteinsäure war nicht nachzuweisen, wohl aber trat eine Dunkelfärbung der Eisenchloridlösung ein, so dass Succinimid durch Pepsin nur in Succinaminsảure úbergefubrt wird.

Trypsin. Der alkoholische Verdampfungsrückstand, mit sehr wenig Wasser aufgenommen, wurde durch verdunnte Salzsaure genau neutralisirt; auf Zusatz von Eisenchlorid und Erwärmung entstand eine tief braunrothe Flüssigkeit, welche beim Kochen keine Ausscheidung gab; eine andere Probe entwickelte mit Kalilauge Ammoniak, so dass ich annehmen möchte, dass auch durch Trypsin keine Abspaltung von Bernsteinsäure aus Succinimid bewirkt, sondern nur Succinaminsäure gebildet wird.

Pty alin ist ohne Einwirkung. 
Versuche mit Histozymen.

Der filtrirte, unter Zusatz von etwas Natriumbicarbonat eingedampfte Digestionsauszug wurde eingedampft, der Rủckstand durch Aetheralkohol vom Succinimid befreit, das Ungeloste nach Verdampfen der Flussigkeit mit 55\% igem Alkohol ausgezogen und nach Neutralisation der Lösung auf die Umsetzungsproducte weitergepruft, wie bei den Enzymen angegeben. Leider störte der Extractionsfarbstoff in der Lossung die Farbenreaction etwas.

Leber. Die mogglichst verdünnte Lösung wurde nach dem genauen Neutralisiren mit Eisenchloridlösung versetzt und bis zum Sieden erhitzt; es entstand hierbei ein dunkelrother, gelatinöser Niederschlag, und da eine Probe, mit starker Kalilauge gekocht, kein Ammoniak entwickelte, durch die entwickelten Dämpfe weder Curcumapapier gebraunt noch rothes Lackmuspapier geblaut wurde, so kann auf Abwesenheit von Succinimid und Succinaminsäure sicher geschlossen werden: es ist durch das Leberhistozym aus Succinimid Bernsteinsäure abgespalten worden, welche auch andererseits beim Ausschutteln der mit Phosphorsảure übersättigten Lòsung mit Aether und Verdampfen desselben in Krystallen erhalten wurde.

$\mathrm{Ni}$ e r e. Die mit Aether und $55 \%$ igem Alkohol ausgezogenen Rúckstände wurden in Wasser gelöst und mit Eisenchloridlósung versetzt: Succinaminsảure und Bernsteinsäure waren nicht nachzuweisen; Nierenhistozym ist obne Wirkung.

Aus den erwähnten Versuchen von Koehne ergibt sich klar, dass bei Futterungsversuchen mit Hunden nur ein sehr geringer Theil des in den Körper eingebrachten Materials denselben unzersetzt passirt, der grỏsste Teil dagegen im Thierkörper in Bernsteinsäure umgewandelt wird und diese selbst dann, wie die Unter-

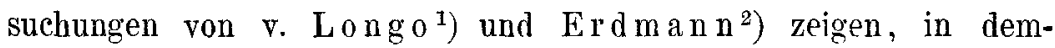
selben vollkommen zerfallt.

Versuche mit pflanzlichen Fermenten.

Maltin, Invertin und Emulsin sind ohne Einwirkung auf Succinimid.

1) Zeitschr. f. physiol. Chemie Bd. 1 S. 214.

2) Ebenda, Anmerkung. 


\section{Succinaminsänre.}

Durch die soeben mitgetheilten, mit Succinimid angestellten Versuche ist erwiesen, dass die meisten Fermente wirkungslos sind, die wirksamen aber, Pepsin und Trypsin, dieses Imid zwar spalten, aber auch nur bis zur Bildung von Succinaminsaiure. So sind denn Prüfungen mit diesen beiden zuletzt genannten Fermenten naturgemäss überflüssig; die mit den ubrigen waren voraussichtlich erfolglos, doch habe ich sie der Vollständigkeit wegen noch angestellt. Ebenso habe ich auch noch Versuche mit Leberhistozym gemacht, um festzustellen, ob Succinaminsäure nicht allein im Entstehungszustande, sondern auch als geschlossenes Molekül zerlegt wird.

Zur Darstellung der Succinaminsäure hat sich mir am meisten folgendes Verfabren bewahrt: Ein Gemisch von 30,0 Succinimid, 48,5 - die äquivalente Menge - Barythydrat und 200,0 friseh ausgekochten destillirten Wassers wurde im Erlenmayer-Kolben mit eingesenktem Thermometer bis $75^{\circ} 24$ Stunden erwärmt; da bei dieser Temperatur sich kein Ammoniak entwickelt, so ist die Einwirkung des Baryumhydroxyds auf Succinamiısäure weniger energisch, als bei Anwendung von Calciumhydroxyd beobachtet.

Vortheilhaft ist es, sich erst Barytwasser frisch darzustellen, da sich immer wieder etwas Baryumearbonat ausscheidet, und in das Filtrat bei $75^{\circ} \mathrm{C}$. das Succinimid einzutragen; dasselbe lost sich sofort, und eine spätere schwache Trubung von Baryumcarbonat kann man unberücksichtigt lassen. Zum Abscheiden der Sáure aus dem succinaminsauren Baryum soll eme geringe Menge des Salzes unzerlegt bleiben; ich entnahm daher der alkalischen Lösung $30 \mathrm{ccm}$, fällte die ubrige Menge mit verdunnter Schwefelsaure genau aus und fugte die zuruckbehaltene Menge $\mathrm{zu}$; das Filtrat wurde vorsichtig concentrirt, in eine grosse Menge mit etwas Aether vermischten Alkohols unter stetem Ruhren in dunnem Strahl eingegossen und die Ausscheidung einige Tage zur volligen Klärung beiseitegestellt. Das mit Aetheralkohol ausgewaschene feine weisse Pulver gab in Wasser gelöst auf Zusatz von Eisenchloridlösung beim Erwärmen eine tief dunkle Färbung, beim Kochen jedoch keine Ausscheidung, so dass das Praparat frei von Bernsteinsäure war. Diese Reaction benutzte ich zur Erkennung der Succinaminsäure fur sich wie auch neben Bernsteinsảure; eine dunkelgefärbte Flussigkeit mit einem gallertigen Niederschlag beim Kochen enthält beide Sauren; 
ist die Flüssigkeit dagegen ùber dem Niederschlag farblos, so war nur Bernsteinsäure zugegen: denn Succinimid gibt mit Eisenchlorid keine Reaction. Da sich bernsteinsaures Natrium, wie mich Versuche lehrten, leicht in 55\% igem Alkohol auflóst, Fluornatrium dagegen wie schon erwähnt, nicht, so bedarf es demgemäss nur des Ausschuttelns der bei den Versuchen erhaltenen Verdampfungsrickstände mit verdunntem Alkohol, um in dieser Lösung nach genauer Neutralisation direct auf Bernsteinsäure prufen zu können; ausserdem verdampfte ich die alkoholische Lösung auf dem Wasserbad, nahm den Rickstand mit wenig Wasser auf und versetzte mit viel Alkohol, filtrirte die Ausseheidung ab, wusch mit Alkohol nach, löste in Wasser und prufte weiter.

Versuche mit thierischen Fermenten.

Ueber Pepsin und Trypsin ist das oben Gesagte zu vergleichen.

Ptyalin war wirkungslos.

Versuche mit Histozymen.

Hier kam es darauf an, in Folge des stark gefärbten Digestionsauszuges, die Eisenreaction deutlich zu machen; es wurde daber der alkalische Verdampfungsruckstand zunaichst mit Aether ausgeschüttelt, um Fett zu beseitigen; den Ruckstand zog ich mit 55\% \% igem Alkohol aus, um Natriumsuccinat in Lossung zu bekommen, und nach mehrmaligem Wiederlösen, Schutteln mit Aether u. s. w. blieb schliesslich ein schwach gelblichgefärbter Ruckstand, welcher, in Wasser gelöst und neutralisirt, mit Eisenlösung gepruft werden konnte.

Leber. Es trat beim Aufkochen der Probeflussigkeit mit Eisenlosung eine starke Dunkelfärbung ein, jedoch keine Fällung, so dass ich annehmen muss, dass ein Theil der Succinaminsaure noch unzersetzt vorhanden ist, ein Theil derselben jedoch in Bernsteinsäure ubergefuhrt, diese aber zweifellos weiterzerlegt ist, weil Succinimid durch Leber in Bernsteinsaure úbergefuhrt wird.

Niere. Beim Erhitzen mit Eisenchloridlosung entstand keine gelatinöse Ausscheidung, so dass eine Bildung von Bernsteinsäure nicht anzunehmen ist.

Versuche mit pflanzlichen Fermenten.

Die pflanzlichen Fermente Invertin, Maltin und Emulsin sind wirkungslos auf Succinamınsäure. 


\section{Dibenzamid.}

Dieses Präparat musste erst dargestellt werden, und sollen nach Barth und Seuhofer ${ }^{1}$ ) in ein Gemisch von 7 Theilen rauchender Schwefelsảure und 4 Theilen wasserfreier Phosphorsaure allmáhlich 7 Theile Benzonitril eingetragen, die Masse durchgeschuttelt und nach einigen Stunden mit viel Wasser versetzt werden. Die Krystalle krystallisirt man aus Alkohol um.

Nach dieser einfach erscheinenden Vorschrift kommt man nicht zum Ziel, denn dem dick-oligen Sáuregemisch sind die vorgeschriebenen Mengen Benzonitril "allmählich" - d. h. doch nach und nach oder in kurzen Pausen - nicht zuzufugen, weil bereits nach der $\mathrm{Zu}$ setzung der Hälfte an Benzonitril die Gesammtmischung steinhart wurde. Den zweiten Versuch mit 50,6 Benzonitril führte ich mit besserem Erfolg so aus, dass ich das Sauregemisch in einen starken Porzellanmórser gab und unter stetem Ruhren ununterbrochen in dünnem Strahl die völlige Menge Benzonitril zufúgte; hierbei trat natúrlich eine Erwärmung ein, welche etwas Benzonitril verfluchtigte, allein das Gemisch konnte bis zuletzt, wenn auch schwierig durch seine steife Consistenz, gut durchgeruhrt werden, - ein „Durchschutteln", wie ich es zuerst nach der Originalvorschrift im Kolben vornehmen wollte, ist geradezu unmogglich.

Die Masse war bald steinhart geworden, blieb bis zum nächsten Tag stehen, liess sich sehr schwer kleiustuckenweis aus dem Mörser bringen, - Porzellanschalen gehen hierbei sicher entzwei; die Stucke zerrieb ich fein, fugte wenig Wasser hinzu und erzielte dadurch nach einiger Zeit ein gleichmässiges, syrupöses Gemisch, welches, noch etwas mehr verdunnt, unter Ruhren in die 300 fache Menge Wassers an Benzonitril eingegossen, schnell eine milchige Flüssigkeit gab, aus sehr feinen Flöckchen von Dibenzamid bestehend. Die vollständige Ausscheidung des Productes dauert mindestens drei Tage; der abfiltrirte, ausgesússte und abgepresste Niederschlag wurde in heissem Alkohol gelost, auf dem Wasserbad mit warmem Wasser bis zur eintretenden Trübung versetzt und die Flussigkeit zur Krystallisation beiseitegestellt; die Mutterlaugen wurden weiterverarbeitet, als noch farblose, geruchfreie Krystalle sich ausschieden.

1) Berichte d. chem. Gesellsch. Bd. 9 S. 975. 
Die Ausbeute betrug gegen $16 \%$ des Benzonitrils, - immer eine geringe.

Unlöslich in Wasser, leicht löslich in Alkohol, zeigt-das Benzdiamid unter dem Mikroskop die der Benzoësaure ähnlichen Fahnengruppen wie auch grosse Nadeln, so dass der sichere Nachweis der Saure erschwert ist, wenn nicht die rhombischen Platten mit den abgesetzten Längsseiten vorhanden sind. Alle Versuchsgemische wurden mit etwas Natriumbicarbonat eingedampft, der Rúckstand mit Wasser wieder aufgenommen, das Filtrat eingeengt, mit Phosphorsaure ubersättigt, mit Aether öfter ausgeschuttelt, diese Lòsung vorsichtig verdampft: das mikroskopische Bild sowie die Eisenreaction gaben mir die Sicherheit von der Gegenwart der Benzoësaure oder Hippursäure.

Versuche mit thierischen Fermenten.

Pepsin. Beim Verdampfen des ätherischen Auszuges auf dem Deckglas zeigten sich federfahnenartige Gebilde, die wohl auf Benzoësäure schliessen liessen; nach dem völligen Eindampfen im Schälchen resultirten neben den grossen, balkenfórmigen Krystallen des Dibenzamids, auch die Tafeln der Benzoësäure; es wird sonach das Dibenzamid durch Pepsin zum Theil in Benzoësảure ubergefuhrt.

Trypsin; eine Verseifung des I)ibenzamids war nicht eingetreten.

Ptyalin ist ohne Einwirkung.

Versuche mit Histozymen.

Leber. Der ätherische Auszug, erhalten aus dem mit Phosphorsaure angesäuerten Rückstand, gab beim Verdampfen tafelförmige Krystalle, die als Benzoësaure angesehen werden konnten; beim Kochen mit starker Lauge entwickelte sich jedoch auch Anmoniak, welches rothes Lackmuspapier bläute; desshalb wurde der Verdampfungsrückstand mit verdünnter Sodalösung längere Zeit digeriit, die Flussigkeit abfiltrirt, mit Phosphorsäure übersättigt, mit Aether ausgeschüttelt und dieser verdampft: es erschienen nunmehr die charakteristischen Krystalle der Benzoësäure mit ihren abgesetzten Längsseiten wie auch gekreuzte Complexe der Hippursäure. Ein anderer Theil der Flussigkeit wurde mit einem Tropfen Eisenchloridlösung versetzt und erhitzt: es entstand eine tief blutrothe Färbung, welche meiner Ansicht nach in diesem Fall nur auf die Gegenwart von Benzoësäure zurùckzuführen ist; eine isabellfarbene Ausscheidung von basischem hippursaurem Eisen trat allerdings nicht ein.

E. Pflüger, Archıv für Physiologıe. Bd 95. 
Es wirkt also das Leberhistozym auf Dibenzamid in gleicher Weise rerseifend ein wie auf Benzamid.

Niere. Durch das Nierenhistozym wird Dibenzamid gleichfalls in Benzoësảure ubergeführt.

Aus den Fütterungsversuchen von Koehne geht hervor, dass das Dibenzamid im Thierkörper unter Bildung von Benzoësäure zersetzt und diese als Hippursäure ausgeschieden wird.

Versuche mit pflanzlichen Fermenten.

Bei den Einwirkungen von Invertin, Maltin und Emulsin waren Verseifungsproducte nicht nachzuweisen.

\section{Disalicylamid.}

Dieses Präparat stellte ich nach der Vorschrift von Schulerud ${ }^{1}$ ) dar durch Ueberleiten von Salzsäuregas úber in einem Verbrennungsrohr vertheiltes und langsam erhitztes Salicylamid. Das erstarrte Umsetzungsproduct, eine gelbliche Masse, wurde mit warmem Wasser so lange verrieben und abgeschwemmt, bis eine Probe mit sehr verdünnter, fast farbloser, durch einige Tropfen Salzsäure angesauerter Eisenchloridlösung keine Blaufärbung mehr gab. Disalicylamid ist in Wasser unloslich, schwer löslich in Aether, ziemlich leicht in heissem Alkohol; die alkoholische Lösung wird durch Eisenchlorid roth gefärbt. Zusatz von Eisenchlorid $\mathrm{zu}$ in Wasser vertheiltem Disalicylamid ruft keine Färbung hervor ${ }^{2}$ ). Den Nachweis von gegebildeter Salicylsaure konnte ich auf gleiche Weise wie bei Salicylamid ausführen, da auch das Disalicylamid in concentrirter Ammoniumacetatlosung nicht loslich ist; doch ich zog vor, die Versuchsgemische zum Theil nach benothigter Zugabe von Natriumearbonat einzudampfen, das Natriumsalicylat durch Wasser auszuziehen, die filtrirte Lösung mit Phosphorsäure zu ubersättigen und den Verdampfungsruckstand auf Salicylsäure zu prufen.

Versuche mit thierischen Fermenten.

Pepsin. Der Aetherrückstand wurde fein zerrieben und mit concentrirter Ammonacetatlösung unter Zusatz einiger Tropfen Salz-

1) Journal f. ¡r. Chemie Bd. 2 H. 2 S. 289.

2) Bellstein Bd. 2 S. 1499. 
Ueber die Verseif barkeit einiger Sảureimide (Diamide) u. Aminsauren etc. 291

säure vermischt, diese Lösung mit Wasser verdünnt, das Filtrat mit Eisenchlorid gepruft - in diesem Falle konnte allein Salicylsäure zugegen fsein - ; es entstand eine tiefblaue Farbenreaction, so dass ich annehmen musste, dass das Disalicylamid durch Pepsin in Salicylsäure ubergefuhrt worden ist. Allein, der Controlversuch mit gekochtem Pepsin gab bei gleichem Verfahren die gleiche, wenn auch scheinbar schwächere Farbenreaction! Nach dieser auffallenden Erscheinung schien es mir möglich, dass die - wenn auch sehr geringe - Menge der in beiden Fällen zugesetzten Salzsäure bereits Salicylsäure abgespaltet haben könnte. Nun hatte ich in den gleichzeitig angestellten Trypsinversuchen beobachtet, dass in beiden Fällen, also auch bei Verwendung von gekochtem Trypsin, Salicylsaure gebildet wurde. Das führte mich denn zu der Vermuthung, die Spaltung werde durch das kohlensaure Natrium veranlasst, welches ja den Trypsin-Versuchsgemischen von vornherein zugesetzt, in den Pepsinversuchen aber in gewohnter Weise zum Neutralisiren benutzt worden war, in dem letzteren Falle nicht ohne Vermeiden eines kleinen Ueberschusses von Soda. In der That zeigte sich, dass auch ganz schwache Sodalösung allein das Disalicylamid spaltet, wenn auch, wie dies bei der Unlöslichkeit des Amids begreiflich ist, nur zu esnem kleinen Theil. Ob das Pepsin gar nicht auf Disalicylamid einwirkt, war nun freilich auch nicht entschieden; ich möchte aber die Einwirkung schliesslich doch als nicht vorhanden bezeichnen, da Aether aus den sauer gebliebenen Versuchsgemischen keinen Fisenchlorid bläuenden Stoff aufnahm.

Mit Trypsın sind nach dem oben Mitgetheilten Versuche unınóglich.

Ptyalin war ohne Einwirkung, weil die Eisenreaction direct vorgenommen wurde und ausblieb.

Versuche mit Histozymen.

Bei der Empfindlichkeit des Disalicylamids Alkalien gegenuber musste ich zunächst feststellen, ob nicht auch das alkalisch reagirende Fluornatrium bereits einwirken konnte. Das ist aber auch bei längerer Digestion offenbar nicht der Fall, ich brauchte also nicht auf die Verwendung des Fluornatriums in meinen wiederholten Versuchen zu verzichten; nur musste der Zusatz von Soda bei den Nierenversuchen fortbleiben; da in den Hauptversuchsgemischen und in den Controlversuchen mit gekochten Organen sich Salicylsäure nachweiscı 
liess, fuhre ich diese auffallende Erscheinung darauf zuruck, dass nach meiner Beobachtung sich das Disalicylamid bereits in $50 \%$ igem Alkohol lóst und demgemäss Eisenchlorid eine Blaufärbung entstehen lassen musste. Die Gemische der neuen Versuchsweise wurden nach der Digestion aufgekocht, das Filtrat bis zum Syrup eingedampft, diesem etwas Ammonacetat zugefugt, die Lösung mit Wasser verdunnt und filtrirt: Disalicylamid muss als unlöslich zuruckbleiben, wahrend, eventuell unter Zusatz von etwas Salzsäure, Fisenchloridlossung im Filtrat Salicylsäure erkennen lassen wurde.

Le ber. Die Filtrate gab ich in sehr verdunnte, mit einigen Tropfen Salzsäure versetzte Eisenchloridlösung: in beiden Versuchen trat nunmehr keine Bläuung oder die Rothfärbung der Salicylreaction ein, so dass ich als erwiesen annehmen muss, dass das Leberbistozym auf Disalicylamid nicht verseifend einwirkt.

$\mathrm{Ni}$ ire. Diese Versuche waren also ohne Zusatz von Sora ausgeführt, und schien mir daher der Erfolg zweifelhaft. Desshalb überraschte es mich, dass bereits das Filtrat vom Hauptversuch mit Eisenchlorid eine kirschrothe Farbung gab, während bei dem Controlversuch diese Reaction nicht eintrat. Im weiteren Verlauf trat die Reaction im Hauptversuch sebr deutlich auf, und nehme ich als erwiesen an, dass Nierenhistozym aus Disalicylamid Salicylsäure abspaltet, jedoch wohl in geringerem Maasse als bei Gegenwart von Soda.

Versuche mit pflanzlichen Fermenten.

Invertin, Maltin und Emulsin sind bei Abwesenheit von Soda wirkungslos.

\section{Phtalimid.}

Auch bei diesen Versuchen traten bedeutende Schwierigkeiten ein, da Phtalimid sowie die Phtalsäure fast gleiche Lösungsverhältnisse und Krystallform zeigen. Die eingetretene Verseifung sollte constatirt werden, wenn Phtalsäure sicher nachgewiesen worden ist; diese würde sich in den Versuchslósungen an Natrium gebunden vorfinden, wenn auch die bisher angewendete Methode eingehalten wird. Landsberg ${ }^{1}$ ) gibt an, dass die Phtalimidsalze beim Behandeln mit Sauren Phtalimid wieder abscheiden; dagegen

1) Liebig's Annalen Bd. 215 S. 181 
Ueber die Verselfbarkett einiger Saureimide (Diamide) u. Aminsauren etc. 293

hat $\mathrm{Koehne}^{\mathrm{1}}$ ) gefunden, dass aus einer wässrigen Phtalimidnatriumlösung - dieses konnte sich wohl beim Eindampfen der Versuchslösung mit Soda bilden - auf Zusatz von Salzsäure bei Zimmertemperatur in $1^{1 / 2}$ bis 2 Tagen, sogar nach ganz kurzer Zeit nicht Phtalımid, sondern Phtalsảure ausgeschieden wird. Nach dieser Beobachtung Koehne's wäre es also absolut unmöglich, genau nachzuweisen, ob die gefundene Menge Phtalsaure während der Einwirkung der Fermente und Histozyme aus Phtalimid entstand oder bereits beim Zerlegen des Phtalimidnatriums durch Phosphorsäure abgeschieden worden ist; aber es bildet sich, wie ein besonderer Versuch zeigte, heim Digeriren und Eindampfen des Phtalimids mit Soda die erwähnte Natriumverbindung nicht; es schied sich aus dem Aetherauszug der mit Phosphorsäure stark angesauerten Ruckstände immer wieder nur Phtalimid aus. Es stand also nichts im Wege, bei den Versuchen selbst oder nachträglich Soda zu verwenden. So wurden denn die Gemische unter Zusatz von etwas Natriumbicarbonat auf dem Wasserbad eingedampft, mit $93 \%$ igem Alkohol, welcher auch phtalsaures Natrium löste, verrieben und mit einer grösseren Menge 24 Stunden unter häufigem Unischutteln digerirt, das Filtrat eingedampft, der Ruckstand eventuell in Wasser gelöst, das Filtrat mit Phosphorsäure ubersätigt, durch Aether ausgeschuttelt und dieser verdampft: der Ruckstand konnte nur Phtalsäure sein.

Versuche mit thierischen Fermenten.

Pepsin. Beim Verreiben des Verdampfungsrúckstandes mit $93 \%$ igem Alkohol entstand bei dem Hauptversuch eine milchige Trubung, aus welcher sich dann eine weisse Ausscheidung absetzte; bei dem Controlversuch trat diese Erseheinung nicht ein, sondern es blieb bei geringer Trubung des Alkohols ein sandiger, sich schnell absetzender Rúckstand. Die auf dem Wasserbad eingedampften Filtrate gaben einen weingelb gefarbten Ruckstand; in wenig Wasser aufgenommen, mit etwas Phosphorsäure versetzt, entstand bei dem Hauptversuch eine sandige Ausscheidung, wäbrend bei dem Controlversuch mit gekochtem Ferment die Lösung klar blieb. Der beim Verdampfen des ersten Aetherauszuges erhaltene Rúckstand wurde nochmals in Aetheralkohol gelöst, und aus dieser Lösung schieden

1) Dissertation. Rostock 1894. 
sich sternförmig gruppirte, faserige Krystalle wie auch lange Nadeln aus, welche denen entsprachen, die aus einer alkoholischen Lösung von reiner Phtalsäure erhalten wurden. Pepsin führt Phtalimid in Phtalsäure über.

Trypsin. Die erhaltenen Krystalle bestanden theils aus Phtalimid - es entwickelte sich beim Kochen mit Lauge Ammoniak -, theils aus Phtalsäure, weil eben ersteres etwas in Wasser löslich ist. Im Controlversuch fand sich nur etwas Phtalimid; auch Trypsin wirkt verseifend.

Ptyalin war ohne Einwirkung.

\section{Versuche mit Histozymen.}

Den Versuchsgemischen wurde nach dem Erkalten, wenn nöthig etwas Natriumbicarbonat bis zur schwach alkalischen Reaction zugefugt und die Flussigkeit sechs Stunden unter öfterem Schütteln stehen gelassen; das weitere Verfahren war wie bei den Fermenten.

Leber. Der Verdampfungsruckstand war gefärbt und wurde nochmals mit Alkohol ausgezogen; beim langsamen Verdunsten resultirten vorherrschend die langen Nadeln der Phtalsäure; da jedoch beim Kochen mit Lauge sich Ammoniak entwickelte, so ist es zweifellos, dass auch Phtalimid zugegen war und durch das Leberhistozym Phtalimid zum gróssten Theil in Phtalsáure übergeführt wird.

Niere. Der ätherische Verdampfungsruckstand war ausserst gering; in dem verbliebenen gelblichen Syrup waren nur spärliche Phtalsäurenadeln zu finden, auch bei erneutem Auflosen in heissem Alkohol und Verdunsten desselben; ich möchte daher annehmen, dass die in der Leber bereits entstandene Phtalsaure während des Passirens der Nieren völlig zerstört wird.

Vergleicht man diese Erscheinungen mit den Resultaten, welche Koehne bei seinen Futterungsversuchen erhielt, sowie mit den Berichten Juvalta's ${ }^{\text {) }}$ ) uber die Zerstöbarkeit der Phtalsảure In Thierkörper, so wird meine Ansicht uber die Wirkuıg des Nierenhistozyms Bestätigung finden.

1) Zeitschr. f phys. Chemie Bd. 12 S. 26. 
Ueber die Verseifbarkeit einiger Saureımide (Diamide) u. Aminsauren etc. 295

Versuche mit pflanzlichen Fermenten.

Invertin und Maltin sind wirkungslos.

Emulsin. Die beiden Versuche unterschieden sich bereits beim Auflósen der attherischen Verdampfungsrückstände in Wasser; bei dem Hauptversuch entstand eine klare, gelbliche Lösung, während bei dem Controlversuch mit gekochtem Emulsin eine milchige Trübung eintrat; beim Ansáuern mit Phosphorsäure und Ausschutteln mit Aether waren wieder getrennte, klare Schichten vorbanden. Bei dem Kontrolversuch war Phtalimid unverkennbar zu constatiren, wäbrend aus dem Hauptversuch sich erst nach einigen Tagen Phtalsäurekrystalle ausschieden; es wirkte also das Emulsin ähnlich wie auf Acetamid und Formanilid verseifend auf Phtalimid ein. -

Zum Schluss lasse ich noch einmal eine Gesammtubersicht der Resultate aus meinen beiden Versuchsreihen folgen. In den horizontalen Reihen fallen sämmtliche Oxalsäurederivate aus, wie an einer anderen Stelle bereits hervorgehoben. In den verticalen Reiben fallen die Enzyme Ptyalin, Invertin und Maltin aus, und es ist wohl mit der grössten Wahrscheinlichkeit anzunehmen, dass auch die nicht untersuchten Enzyme dieser Gruppe die Säureimide nicht zerlegen. Das verschiedene Verhalten der anderen Enzyme lässt sich einstweilen wobl noch nicht in ein einfaches Bild zusammenfassen; der Wirkungsbezirk eines jeden Fermentes wird sich erst

\begin{tabular}{|c|c|c|c|c|c|c|c|c|}
\hline $\begin{array}{l}\text { Saureamide und -Ani- } \\
\text { lide, ImIde und Amin- } \\
\text { sauren }\end{array}$ & 寻 & : & 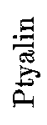 & 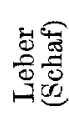 & 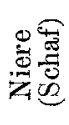 & 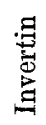 & 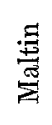 & $\underset{\text { 超 }}{\stackrel{\Xi}{\Xi}}$ \\
\hline Formamid & - & - & - & + & + & - & - & - \\
\hline Acetamid. . . & - & + & - & + & I & - & E & $\overline{+}$ \\
\hline Oxamid. . . & 一 & - & - & $\ldots$ & 一 & $\ldots$ & 一 & - \\
\hline Succinamid . . . . & - & - & - & + & - & - & - & $\ldots$ \\
\hline Benzamid. . . . & - & - & - & + & + & $\ldots$ & - & - \\
\hline Salıcylamid . . . . & - & - & - & - & + & - & - & - \\
\hline Formanilid . . . & + & + & - & + & + & - & - & + \\
\hline Acetanılid. . . . & + & + & - & - & - & - & - & - \\
\hline Oxanilid . . & - & - & - & 一 & - & - & - & - \\
\hline Benzanilid . . & - & - & - & - & + & - & - & - \\
\hline Oxaminsaure . . . & - & - & - & - & - & - & - & - \\
\hline Succinimid . . . & + & + & - & + & - & - & - & - \\
\hline Succinaminsaure . . & - & - & - & + & - & - & - & - \\
\hline Dibenzamid . . & + & - & - & + & + & — & 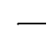 & - \\
\hline Disalicylamid . . . & - & - & - & - & + & - & - & - \\
\hline Phtalimid. . . & + & + & - & + & + & - & - & + \\
\hline
\end{tabular}


296 M. Gonnermann: Ueber die Verseifbarkeit einiger Säureimide etc.

nach Prüfung an den verschiedenartigsten Körpern bekannter Constitution feststellen lassen ${ }^{1}$ ); hervorgehoben könnte wohl die Leistungsfähigkeit der Leber werden; ihr kommt die der Niere sehr nahe, doch ist Beides im Allgemeinen bekannt aus früheren Untersuchungen.

1) Vgl. hierzu die Arbeiten von Fischer, Zeitschr. f. phys. Chem1e Bd. 26 S. 71. 1898. - Chem. Ber. Bd. 27 S. 2992. 